\title{
Guía "De regreso al trabajo", necesidad empresarial para la adecuada implementación de los procesos de rehabilitación, reincorporación y reubicación laboral dentro del sistema de gestión de seguridad y salud en el trabajo*
}

"Return to work" guide, business necessity for the adequate implementation of the rehabilitation, reincorporation and labor relocation processes within the workplace safety and health management system

Guia de "de volta ao trabalho" necessidade empresarial para a adecuada implementação dos processos de reabilitação, reincorporação e relocalização trabalhista dentro do sistema de gestão de segurança e saúde no trabalho

Ericka Alexandra Jiménes Rodrígue***

Corporación Minuto de Dios, Colombia

Jeimy Angélica Camberos Bustos ***

Profesional compañia de seguros / administradora de riesgos laborales

Citar como: Jiménez Rodríguez, E. A., y Camberos Bustos, J. A. (2019). Guía "De regreso al trabajo", necesidad empresarial para la adecuada implementación de los procesos de rehabilitación, reincorporación y reubicación laboral dentro del sistema de gestión de seguridad y salud en el trabajo. SIGNOS - Investigación en sistemas de gestion, 11(1), 87-98. DOI: https://doi.org/10.15332/s2145-1389.2019.0001.05

* Artículo de resultado de investigación.

** Especialista en Higiene y Salud Ocupacional, especialista en Gestión de Proyectos, ingeniera industrial. Línea de investigación: Gestión Social, Participación y Desarrollo Comunitario, semillero ATARAXIA. Corporación Minuto de Dios, Colombia. Correo electrónico: ejimen17@uniminuto.edu.co. ORCID: https://orcid.org/0000-0003-3938-9335

*** Magíster en Prevención de Riesgos Laborales, especialista en Gerencia en Salud Ocupacional, terapeuta ocupacional. Profesional compañía de seguros/administradora de riesgos laborales, Colombia. Correo electrónico: jeimy.camberos@positiva.gov.co. ORCID: https://orcid. org/0000-0002-9029-502X 


\section{RESUMEN}

La integración sociolaboral es considerada como el objetivo inmediato de la rehabilitación profesional y final de la rehabilitación integral. Su intervención está orientada a la recuperación y desarrollo de la capacidad de la persona que ha sufrido alguna disminución de su capacidad para ejercer una función o un cargo, o para desempeñar funciones productivas en diferentes contextos, con base a los estándares de calidad establecidos por el sector productivo. Los procesos de rehabilitación, reincorporación o reubicación laboral son pobremente conocidos por los empresarios y la información respecto a estos es escasa y poco estandarizada, lo que dificulta su éxito final. Por lo tanto, a través de esta investigación cualitativa, desarrollada mediante una revisión del estado del arte sobre el tema, se buscó construir un documento técnico-instruccional y pedagógico, denominado "De regreso al trabajo", que permitirá a los encargados del sistema de gestión de seguridad y salud en el trabajo, jefes de talento humano, gerentes y demás personas involucradas en el proceso, aplicar de manera efectiva dichas estrategias para reducir las pérdidas de tiempo por incapacidad temporal y al trabajador retornar al entorno laboral de manera exitosa, manteniendo su papel como actor productivo de la sociedad.

Palabras clave: rehabilitación, reincorporación, reubicación, salud ocupacional, seguridad y salud en el trabajo.

\section{ABSTRACT}

The socio-occupational integration is considered as the professional rehabilitation's immediate objective and the integral rehabilitation's goal. Its intervention is oriented to the recovery and development of the capability of the person who has suffered some capacity decrease to exercise a function or a position, to perform productive functions in different contexts, based on the quality standards established by the productive sector; the rehabilitation, reincorporation or labor relocation processes are poorly known by employers and information about them is scarce and little standardized, which difficults their final success. Therefore, through this qualitative research, developed through a review of the matter's state of the art, we look up to build a technical document - instructional and pedagogical, called "back to work", which will allow those in charge of the System Occupational Health and Safety Management, Human Resource Managers, Executives and others involved in the process, effectively apply these strategies to reduce time losses due to temporary disability and for the worker to succesfully return to the work environment, maintaining its role as a society's productive actor.

Keywords: Rehabilitation, reincorporation, relocation, occupational health, safety and health at work.

\section{RESUMO}

A integração sociolaboral é considerada como o objetivo imediato da reabilitação profissional e final da reabilitação integral. Sua intervenção está orientada à recuperação e desenvolvimento da capacidade da pessoa que tem sofrido alguma diminuição da sua capacidade para exercer uma função ou um cargo para desempenhar funções produtivas em diferentes contextos, baseado nos padrões de qualidade estabelecidos pelo setor produtivo. Os processos de reabilitação, reincorporação ou relocalização laboral são pobremente conhecidos pelos empresários e a informação respeito a estes é escassa e pouco padronizada, o qual dificulta seu sucesso final. Por tanto, através desta pesquisa qualitativa desenvolvida mediante uma revisão 
do estado da arte sobre o tema, buscou-se construir um documento técnico-instrucional e pedagógico denominado "De volta ao trabalho", que permitira aos encarregados do sistema de gestão de segurança e saúde no trabalho, chefes de talento humano, gerentes e demais pessoas envolvidas no processo aplicar de maneira efetiva estas estratégias para reduzir as perdas de tempo por incapacidade temporal e ao trabalhador retornar ao entorno laboral de forma bem-sucedida, mantendo seu papel como ator produtivo da sociedade.

Palavras chave: reabilitação, reincorporação, relocalização saúde ocupacional, segurança e saúde no trabalho.

\section{INTRODUCCIÓN}

Debido a la industrialización y tecnificación de los procesos productivos que actualmente se desarrollan en la industria, el estrés cotidiano y el ritmo de vida que dificulta establecer hábitos saludables de vida ha incrementado la ocurrencia de enfermedades y accidentes de origen laboral o común, que generan limitaciones al desempeño laboral y de las actividades básicas cotidianas de los trabajadores de todos los sectores económicos, condiciones que se hacen relevantes por datos señalados por la Organización Mundial de la Salud y el Ministerio de Salud colombiano.

La Organización Mundial de la Salud (2017) estima que alrededor del $15 \%$ de la población mundial son personas con discapacidad y que tal proporción está en aumento. El Ministerio de Salud indica que el 28.3\% de las personas con discapacidad refieren que su discapacidad es principalmente consecuencia de alguna enfermedad general, el $11.3 \%$ indica que es consecuencia de algún accidente y un $9.9 \%$ señala que es principalmente debido a alteraciones genéticas o hereditarias (Ministerio de la Protección Social, 2015, p. 3).
En lascifras históricas de la Federación de Aseguradores Colombianos (Fasecolda) sobre accidentalidad, cobertura de trabajadores, incapacidad permanente, enfermedad profesional e invalidez, entre los años 2000 y 2011, se muestra un incremento, así:

En las tasas de accidentalidad (para el 2015 una tasa de 7.5 por cada 100 trabajadores) y enfermedad laboral (para el 2011 de 99.24 por cada 100.000 trabajadores), y el pago de prestaciones asistenciales por riesgos laborales asciende a 591.000 millones de pesos colombianos; y 639.721 millones por pago de prestaciones económicas (Fasecolda, 2016, p. 1).

Es por esto que actualmente en los entornos laborales se ha incrementado la necesidad de realizar un mejor seguimiento a las condiciones de la salud de los trabajadores, y a su vez el diseño de estrategias para preparar el retorno exitoso de estos trabajadores con limitaciones temporales o definitivas, para evitar así posibles pérdidas económicas o la afectación de la calidad de sus procesos productivos por el bajo aporte de los mismos.

Para que dicho retorno sea posible, se requiere que las empresas diseñen e implementen procesos de rehabilitación, reincorporación o reubicación laboral de los trabajadores con pérdida de capacidad laboral; sin embargo, por el escaso conocimiento y material de apoyo que se tiene sobre el tema, es difícil que el nivel gerencial, los encargados de seguridad y salud en el trabajo y las entidades colaboradoras en el proceso, impartan directrices claras que permitan alcanzar el objetivo de este retorno laboral.

Estos procesos de reintegración laboral han sido apoyados desde la parte legal por instancias como la Organización Internacional del Trabajo (OIT), en sus estrategias y plan de acción para la inclusión de la discapacidad 2014-2017, y la normatividad legal colombiana. El Ministerio de la Protección Social, desde 
el 2005, estableció dentro del Plan Nacional de Salud Ocupacional 2013-2021, línea III, Objetivo 3.1.3, "la directriz de fortalecer los mecanismos de protección de trabajadores con debilidad manifiesta, en los programas presentes de las diferentes administradoras de riesgos laborales en Colombia" (Ministerio del Trabajo, 2014, p. 36). En el año 2010 el Ministerio del Trabajo expide el Manual de Procedimientos para la Rehabilitación y Reincorporación Ocupacional de los Trabajadores en el Sistema General de Riesgos Laborales, que es actualizado en el año 2012, donde se dan algunos lineamientos para el reintegro de trabajadores con pérdida de su capacidad laboral, el cual no ha sido ampliamente difundido en las empresas. Así mismo, se estableció en la Resolución 1111 de 2017 estándar 3.1.6 "que es tarea de las empresas tener un procedimiento claramente establecido y efectuar un seguimiento adecuado al reintegro de sus trabajadores para que identifiquen y ejecuten las opciones de reintegro de acuerdo con el proceso productivo de la empresa" (Ministerio del Trabajo, 2017, p. 28).

A pesar de estos lineamientos y teniendo en cuenta la información de cifras históricas sobre accidentalidad, cobertura de trabajadores, incapacidad permanente, enfermedad profesional e invalidez para antes del año 2012 establecidas por Fasecolda (2016, p. 1), y el consolidado de estadísticas de accidentes y enfermedades laborales 2015-2016 del Fondo de Riesgos Laborales, es posible evidenciar el poco o inexistente conocimiento y aplicación de dichos lineamientos, reflejándose esto en la ausencia de programas de rehabilitación laboral en las empresas, lo cual se pueden ver en la falta de estudios de casos referenciados en empresas antes del 2014, en los reprocesos durante la atención de los trabajadores, en los incontables pagos por incapacidades medicas prolongadas y en los extensos tiempos de espera para acceder a prestaciones asistenciales que permitan una continuidad y pronta recuperación (Ministerio del Trabajo, 2010).
Igualmente, durante las evaluaciones anuales a los sistemas de gestión de seguridad y salud en el trabajo que efectúan las administradoras de riesgos laborales, se refleja el desconocimiento de los encargados de recursos humanos y líderes de seguridad y salud en el trabajo de los tramites frente a las entidades de salud y la verdadera función de cada profesional de salud en el proceso de rehabilitación, reincorporación o reubicación laboral del trabajador, de acuerdo a los análisis realizados en el panel Retos de la cobertura de ITP en Colombia (Fasecolda, 2016, p. 1).

Por las situaciones anteriormente expuestas, se planteó la necesidad de desarrollar un proyecto de investigación que permitiera el diseño de una herramienta de socialización de los procesos de rehabilitación y reincorporación laboral de los trabajadores en el sistema general de riesgos laborales para gerentes y encargados del sistema de gestión de seguridad y salud en el trabajo, que sirva de consulta y facilite la gestión a nivel empresarial de dicho proceso, permitiendo así el retorno temprano y exitoso del trabajador al entorno laboral y la disminución de los costos generados a las empresas $\mathrm{y}$ al sector asegurador.

\section{METODOLOGÍA}

Para el logro del objetivo planteado en la investigación, se llevó a cabo un proceso de tipo cualitativo, de carácter descriptivo, basado en una revisión documental que permitió establecer el estado del arte de los documentos, protocolos, guías o modelos existentes para instaurar los procesos de rehabilitación, reubicación o reincorporación laboral, como base para diseñar un elemento de divulgación tipo cartilla, que facilite implementar estrategias de rehabilitación laboral a gerentes, jefes de talento humano y líderes de seguridad y salud en el trabajo de todo tipo de empresas 
Guía "De regreso al trabajo", necesidad empresarial para la adecuada implementación de los procesos de rehabilitación, reincorporación y reubicación laboral...

Figurara 1. Proceso de diseño de la guía "Retorno al trabajo"

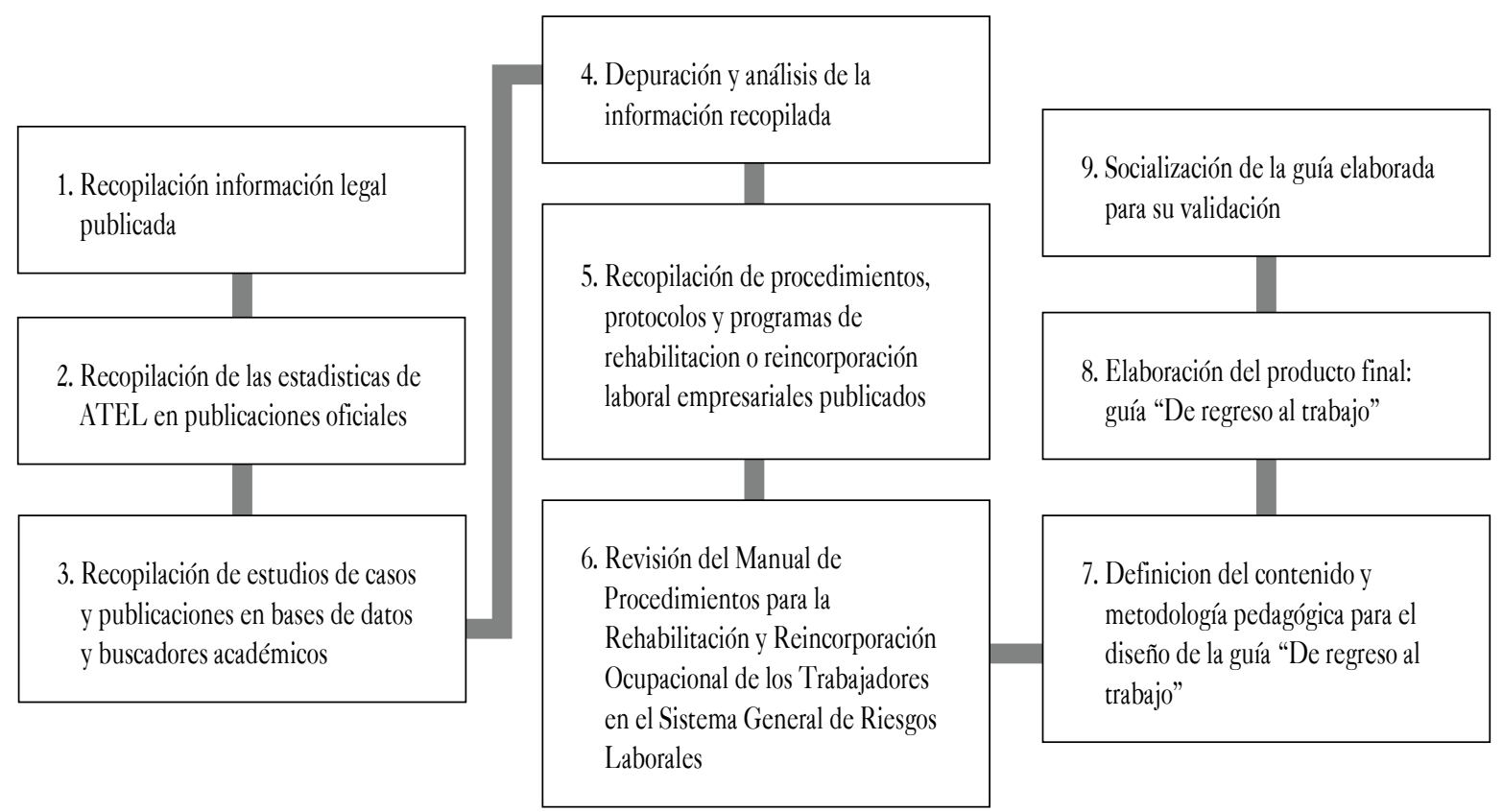

Fuente: elaboración propia (2018).

colombianas. Dicha herramienta se construyó bajo el proceso descrito en la Figura 1.

\section{Proceso de diseño de la guía "Retorno al trabajo"}

1. La información legal se obtuvo a través de la recopilación de la normatividad vigente en el país, establecida en el normograma de la discapacidad en la República de Colombia, que corresponde a los convenios internacionales, leyes, resoluciones, decretos y circulares relacionados con la discapacidad laboral revisando cada uno de los artículos de las mismas, para establecer las responsabilidades de los empleadores, los trabajadores, las entidades prestadoras de salud, las administradoras de riesgos laborales y las juntas de calificación de invalidez, que tengan relación con el proceso de rehabilitación, reubicación o reincorporación.

2. Desde el estudio de casos médicos, se identificó la literatura existente mediante la búsqueda en las bases de datos de Scopus, Scielo y en el buscador académico Google Scholar, a través de las palabras: rehabilitación [rehabilitation], reincorporación [reincorporation], reubicación [relocation], rehabilitación laboral [labor rehabilitation] en español e inglés, y tomando como parámetro de inclusión los libros y artículos escritos a partir de 1985, referentes a los procesos de rehabilitación, reubicación o reincorporación laboral de los trabajadores luego de haber sufrido un accidente 0 una enfermedad laboral o común.

3. Desde la parte médico-técnica, se compilaron las funciones específicas en este campo de las profesiones que juegan un papel importante en este proceso mediante la ejecución de actividades de diagnóstico, pronóstico funcional, pronóstico laboral y plan de tratamiento, como lo son la terapia ocupacional, la fisioterapia, la psicología, la ortopedia y la medicina laboral, entre otras, de acuerdo con los estudios de casos en diferentes procesos, para determinar los aportes desde cada ciencia a los procesos de rehabilitación funcional, ocupacional y 
laboral así como las recomendaciones generales en casos repetitivos.

4. En este paso se tomaron los artículos, libros o tesis, y se revisó a través de la lectura, cuál sería la información analizada por cada uno que serviría como sustento técnico de la guía "De regreso al trabajo".

5. Se recopilaron a través de la plataforma Google Scholar, procedimientos, protocolos y programas de rehabilitación o reincorporación laboral empresariales publicados. Los seleccionados corresponden a entidades como la Alcaldía de Pereira, la Universidad Tecnológica de Pereira, servicios de la Unidad de Medicina Laboral de Colmédicos S. A. sede de Patio Bonito (Medellín), la Gobernación de Norte de Santander, el Instituto Colombiano de Bienestar Familiar, las Fuerzas Militares de Colombia, la Universidad de Santander, los protocolos de la AXA Colpatria, y la tesis denominada "Descripción del proceso de reincorporación laboral, con base al manual de procedimientos en trabajadores de un ingenio del Valle del Cauca período 2012 a 2015”.

6. Se efectúo la revisión del Manual de Procedimientos para la Rehabilitación y Reincorporación Ocupacional de los Trabajadores en el Sistema General de Riesgos Laborales, analizando los lineamientos propuestos para la rehabilitación integral de la población trabajadora.

7. Con los datos obtenidos a partir del análisis de la información de la revisión bibliográfica, se inició el esbozo del documento final, el cual se estructuró con el marco teórico y los lineamientos de proceso de rehabilitación establecidos en el manual indicado, teniendo en cuenta los ítems de alcance, responsabilidades, desarrollo del programa, actividades por fase, puntos de control para la toma de decisiones, registros por fase y cuadros de control.
8. El documento final corresponde a una guía práctica de aplicación que permite direccionar el diseño de programas de reubicación y reintegro laboral a la luz de la legislación vigente.

9. Esta herramienta es validada con profesionales del área de seguridad y salud en el trabajo y profesionales de la salud para su posterior divulgación.

\section{RESULTADOS Y DISCUSIÓN}

Acorde a los resultados obtenidos de la revisión documental de protocolos, guías y normativas, principalmente del Manual Guía de Procedimientos de Rehabilitación y Reincorporación Ocupacional de los Trabajadores en el Sistema General de Riesgos Profesionales, se pudo establecer que la readaptación o reintegro laboral incluye:

El conjunto de procesos que van desde la evaluación, orientación, hasta la rehabilitación profesional propiamente dicha, que comprende todas las intervenciones de orden científico, técnico, educativo-formativo, y sociales que conduzcan a la persona con discapacidad a la obtención y conservación de un trabajo concordante y acorde con sus capacidades residuales (Ministerio del Trabajo, 2010, p. 17).

Dicho proceso de rehabilitación ha sido definido como:

El conjunto de acciones sociales, terapéuticas, educativas y de formación; de tiempo limitado; articuladas y definidas por un equipo multidisciplinario, y que involucran al trabajador, como sujeto activo de su propio proceso, a la familia, a la comunidad laboral y a la comunidad social para generar cambios en el trabajador y en su entorno, que le permitan la reincorporación ocupacional y el cumplimiento de los objetivos trazados que apunten a experimentar una buena calidad de vida (Ministerio del Trabajo, 2010, p. 17). 
Y a pesar de que la legislación colombiana ha reglamentado con diferentes dispositivos la obligatoriedad de la reincorporación laboral cuando la persona con invalidez ha recuperado parte de su capacidad, y ha buscado proteger a los trabajadores cuando se produce un despido injustificado en razón de alguna discapacidad, en el ámbito laboral dichos procesos se han implementado de manera empírica de acuerdo con los recursos técnicos y financieros de las empresas, sin tener en cuenta las necesidades de los trabajadores, ni los manuales, guías o protocolos diseñados por las entidades administradoras de riesgos laborales y el Ministerio del Trabajo.

Muestra de ello, es el escaso conocimiento y aplicación que se le da a la herramienta oficial diseñada por un grupo interdisciplinario del Ministerio de la Protección Social, promulgada en 2010 y actualizada en 2012, denominada Manual de Procedimientos para la Rehabilitación y Reincorporación Ocupacional de los Trabajadores en el Sistema General de Riesgos Laborales (Ministerio del Trabajo, 2010), donde se establecen los procedimientos en las siguientes etapas:

- Etapa I: Identificación de los casos a ingresar en el programa de rehabilitación.

- Etapa II: Evaluación del caso.

- Etapa III: Desarrollo del plan de rehabilitación.

- Etapa IV: Información y retroalimentación del SGRP.

Actualmente se han propuesto modificaciones al respecto por parte del Ministerio del Trabajo, las cuales están en estudio.

Sin embargo, durante el análisis de los documentos recopilados, fue posible evidenciar que dicho manual fue construido con lenguaje técnico, de difícil comprensión por los encargados del proceso al interior de las empresas, por lo que es necesario llevarlo a un lenguaje administrativo, de fácil aplicación y que permita de paso dar cumplimiento con los requerimientos legales relativos al tema.

Por otro lado, la revisión documental permitió establecer que, desde la práctica y la ejecución de actividades diarias, las administradoras de riesgos laborales han establecido estadísticas propias de accidentalidad y enfermedad laboral, las cuales han sido remitidas al Ministerio de la Protección Social, las que a su vez son recopiladas por Fasecolda y divulgadas a través de la página "Informe de coyuntura a agosto de 2013", que ha permitido la creación de programas de prevención y promoción de la salud, así como programas de rehabilitación integral, apoyándose en las fases propuestas por el manual, buscando de esta manera controlar los efectos de dichos siniestros (Fasecolda, 2016, p. 3).

Dentro del análisis de resultados obtenidos, también se encontró que las administradoras de riesgos laborales tienen como directriz común enfocar dichos programas de rehabilitación integral para el tratamiento temprano, debido a la frecuencia de ocurrencia y grado de severidad de lesiones como (ARL Colpatria, 2015, p. 3):

- Amputación de cualquier segmento corporal, independientemente de su extensión.

- Fractura de huesos (vertebras, fémur, tibia, peroné, húmero, radio, cubito).

- Quemaduras de segundo y tercer grado.

- Lesiones del sistema nervioso central y periférico tales como:

- Trauma craneoencefálico.

- Trauma raquimedular.

- Polineuropatías.

- Lesiones severas de plexos, raíces, nervios periféricos o en mano, entre otras: 
- Aplastamiento.

- Quemaduras.

- Avulsiones.

- Rupturas tendinosas o de nervios.

- Lesiones oculares que comprometan la agudeza 0 campo visual.

- Lesiones que comprometan la capacidad auditiva.

- Enfermedad laboral.

- Incapacidad temporal que supere los tiempos previstos para el diagnóstico específico.
El análisis de la información recopilada nos lleva a establecer una herramienta didáctica que permita mejorar la comprensión y aplicación por parte de las empresas del programa de reubicación y readaptación laboral, facilitando así el reintegro efectivo de trabajadores que presentan restricciones médico-laborales, temporales o definitivas, generando a futuro casos exitosos, que permitan a estos individuos mantener su rol productivo en la sociedad. Para ello, se diseñó la guía didáctica "De regreso al trabajo", que responde a las necesidades evidenciadas en la investigación y que consta de cinco capítulos, que se presentan en la Figura 2.

Fiǵura 2. Contenido de la guía "De regreso al trabajo"

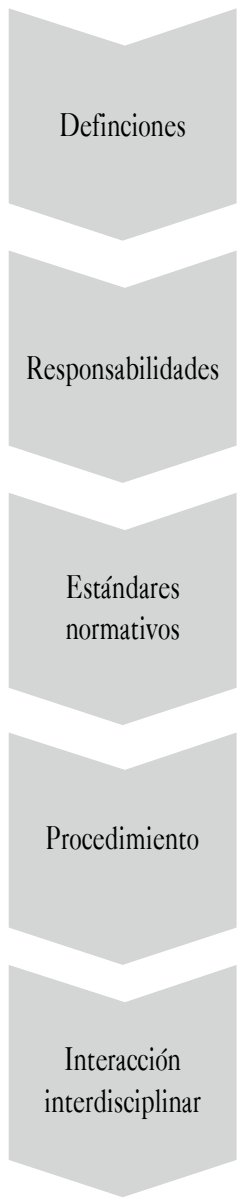

Comprende las definiciones de cada uno de los programas mencionados y la justificación de cada uno para la sociedad, las empresas y los profesionales del área de seguridad y salud en el trabajo.

Se presentan las funciones a ejercer por cada uno de los profesionales que apoyan el proceso, los trabajadores, la alta dirección en cabeza de los gerentes o representantes legales, los encargados del sistema de gestión de seguridad y salud en el trabajo y las juntas de calificación de invalidez. dar cumplimiento a dichos procesos.

Establece a manera de flujogramas los pasos requeridos en cada etapa, para que las empresas puedan desarrollar procesos de rehabilitación, reincorporación y reubicación laboral exitosos.

Refiere las funciones que tiene cada una de las profesiones del área de salud y área técnica en los procesos, como grupo asesor de dicho proceso.

Fuente: elaboración propia (2018). 
La herramienta diseñada se clasifica en una guía de aplicación de los conceptos revisados: "La utilidad más cercana es matizar un contenido difícil que requiere ser contextualizado. Cumple una función de activar potencialidades, trabajar empíricamente y también, para asimilar a su realidad lo trabajado en la parte teórica" (Fundar, 2001, p. 11). La guía de aplicación "De regreso al trabajo", busca apoyar al encargado de SG SSST a decidir qué, cómo, cuándo y con ayuda de qué, aplicar los contenidos existentes en materia de rehabilitación y reintegro laboral con el fin de mejorar el aprovechamiento del tiempo disponible y maximiza su aplicación. Entre las características de una guía se tiene (Universidad Autónoma Chapingo, 2009, p. 3):

- Ofrece información acerca del contenido y su relación con el programa de estudio de la asignatura para el cual fue elaborada.

- Presenta orientaciones en relación con la metodología y enfoque de la materia de estudio. Presenta también instrucciones acerca de cómo construir y desarrollar el conocimiento (saber) y aplicarlo al entorno laboral.

Esta herramienta pretende apoyar la labor del encargado de liderar los programas de rehabilitación y reincorporación laboral brindando estrategias y lineamentos para adelantar acciones como el seguimiento del proceso de intervención temprana del trabajador lesionado para recuperar su funcionalidad en menor tiempo, reintegro, reubicación o reconversión laboral de trabajadores con pérdida de capacidad laboral temporal o definitiva, luego de la calificación por médicos laborales o juntas de calificación de invalidez.

El uso de esta herramienta permite dar cumplimiento a la normatividad legal, evita la pérdida de tiempo en el proceso de rehabilitación de trabajadores lesionados por desconocimiento de las directrices de actuación para las empresas y facilita de esta manera el trabajo del área de recursos humanos, los líderes de seguridad y salud en el trabajo y los representantes legales.

Los procesos de rehabilitación, reincorporación y reubicación, cuentan con un fundamento legal establecido en la Ley 100 de 1993, artículo 153 numeral 3, artículo 162; la Ley 776 del 2002 y el Decreto Ley 1072 del 2017, que establecen como responsabilidad del empleador el establecimiento del programa y el cubrimiento de los procesos asistenciales y de rehabilitación integral por parte de las ARL, a través de las IPS, a los trabajadores que sufran accidentes de trabajo o se les diagnostiquen enfermedades profesionales. Igualmente, por el Decreto 1072 del 2015, en el cual se debe planificar, implementar y hacer seguimiento a los programas de rehabilitación de los trabajadores por parte de las empresas y las obligaciones por la junta de calificación de invalidez en el proceso de calificación, y por último, es importante resaltar lo establecido en la Resolución 1111 del 2017, estándar 3.1.6, donde designa la obligación a las empresas, independientemente de su actividad económica, de diseñar un procedimiento para el adecuado reintegro de sus trabajadores y que efectúen un seguimiento al mismo que garantice su eficacia, para lo cual el Ministerio del Trabajo se encuentra diseñando un proyecto de ley.

Dentro de los documentos revisados, se encontró escasa evidencia de protocolos, procedimientos y documentos que apunten al cumplimiento de este marco legal ya que, como se ha mostrado durante este artículo, el último manual oficial fue actualizado en 2012 y desde esta fecha en Colombia ha existido un crecimiento tangencial de la legislación aplicable en el campo de la reubicación y reintegro laboral para las empresas, y los procedimientos encontrados en esta materia son insuficientes, comparándolos con el número de empresas legalmente establecidas, por lo que el diseño de protocolos, guías o manuales que contemplen estos cambios legales se hace indispensable. 
Teniendo en cuenta que el manual mencionado presenta sus conceptos y directrices utilizando lenguaje técnico de poco manejo por el personal encargado del sistema de gestión de seguridad y salud en el trabajo, esto podría ser la causa del escaso diseño de programas de reubicación y reintegro laboral encontrado en las empresas. Por ello, la guía "De regreso al trabajo", fruto de la recopilación de la información técnico-legal elaborada, presenta de manera didáctica los lineamientos técnicos necesarios para poder implementar de manera exitosa programas de rehabilitación, reubicación 0 reincorporación laboral en todo tipo de empresas, independientemente de su actividad económica, planteando un modelo didáctico, amigable y de fácil comprensión para el personal administrativo empresarial.

Esta guía presenta los contenidos de forma gráfica, utilizando herramientas que estimulan el aprendizaje visual y la fácil compresión de conceptos técnicos permitiendo al encargado del SG SST, mediante pasos sencillos, concebir las acciones necesarias para lograr un proceso de readaptación eficiente del trabajador a su entorno laboral, lo cual complementa y busca una aplicación práctica del Manual de Procedimientos para la Rehabilitación y Reincorporación Ocupacional de los Trabajadores en el Sistema General de Riesgos Laborales.

Dentro de la guía, se da alcance a la definición de los términos rehabilitación, reubicación, reintegro y reincorporación laboral, ya que comprende las definiciones de cada uno de estos y los beneficios de su aplicación para la sociedad, las empresas y los profesionales del área de seguridad y salud en el trabajo, información que no se evidenció en los estudios de casos revisados.

En el manual y demás documentos técnicos revisados, solo se presentan los pasos a desarrollar en el proceso de reubicación o reintegro laboral, sin embargo, no se encuentran definidas las acciones administrativas necesarias para su cumplimiento, mientras que en la guía "De regreso al trabajo", se exponen las funciones a ejercer por cada uno de los profesionales que apoyan el proceso, los trabajadores, la alta dirección en cabeza de los gerentes o representantes legales, los encargados del sistema de gestión de seguridad y salud en el trabajo y las juntas de calificación de invalidez, permitiendo que cada uno de los actores involucrados en el proceso conozca sus responsabilidades, para comprometerse en su ejecución.

Este proceso de reubicación o reintegro laboral ha dejado de ser optativo para volverse una obligación legal para las empresas, tanto así que ha sido incluido como un objetivo prioritario en el Plan Nacional de Seguridad y Salud en el Trabajo 2013-2021, y se han generado proyectos de ley para regular el diseño de este programa; sin embargo, esta normatividad es desconocida por las empresas, lo cual se evidencia en los escasos estudio de caso encontrados durante la revisión bibliográfica; esta información se presenta de manera actualizada dentro de la guía diseñada a manera de lista de verificación que permite de manera práctica una comprobación del cumplimiento legal en esta materia.

El manual muestra en forma lineal cada una de las etapas concebidas en el proceso de reubicación o reintegro laboral, estableciendo: el objetivo, la actividad y el responsable, pero no presenta los criterios para la toma de decisiones frente a las variaciones del proceso, por lo que los procedimientos revisados, al ser copia de estos, manejan un lenguaje técnico que dificulta la aplicación por parte de profesionales que no pertenecen al área de la salud, lo cual interfiere en su efectividad. Adicionalmente, estos procedimientos se manejan de manera aislada y no se articulan con otros programas de prevención y vigilancia epidemiológica diseñados por la empresa, para dar cumplimiento al sistema de gestión de seguridad y salud en el trabajo; estas deficiencias pretenden ser solucionadas dentro de la guía, utilizando flujogramas para mostrar de manera visual 
la línea de pasos y acciones necesarios para interactuar dentro de las etapas, permitiendo que el proceso de rehabilitación y reincorporación laboral fluya hacia el objetivo de la rehabilitación integral del trabajador, alineado a los objetivos establecidos por el manual y buscando de esta manera que este programa sea aplicado de manera práctica por los actores involucrados.

Considerando que dentro del proceso de rehabilitación y reincorporación laboral participan diversas profesiones como medicina general, medicina laboral, terapia ocupacional, terapia física, psicología, medicina física, trabajo social, fonoaudiología y ortopedia, entre otras, como grupo asesor de dicho proceso, y que dentro de la documentación revisada no se hace explícita la interacción de estos profesionales en la toma de decisiones en cada una de las etapas de este proceso, se consideró necesario establecer un último capítulo que le permita al responsable del sistema de gestión de seguridad y salud en el trabajo, conocer las funciones específicas que realiza cada profesional dentro del programa, permitiendo la asertividad del proceso de reubicación y reincorporación laboral y evitando los retrasos que de manera temporal 0 definitiva puedan sufrir los trabajadores que hayan sufrido un accidente 0 enfermedad de origen común 0 laboral, durante su retorno al ámbito laboral.

\section{CONCLUSIONES}

La construcción del documento se desarrolló en varias etapas: las primeras constituyen la definición del estado del arte, mediante la recopilación y análisis de los documentos existentes en las bases de datos Scopus, Scielo y la plataforma Google Scholar, escogiendo aquellos que explicaran los procesos en mención, los procedimientos técnicos existentes, las funciones y responsabilidades de los profesionales de la salud involucrados en el proceso durante las fases de diagnóstico, pronóstico, tratamiento y plan de reintegración del trabajador que ha sufrido un accidente 0 enfermedad que afecte su desempeño laboral; así mismo, se efectúo una revisión de la normatividad legal aplicable al tema.

En la segunda etapa se organizó la información recopilada de los diversos modelos, guías, protocolos y procedimientos revisados de forma coherente, estableciendose cinco capítulos donde se abordaron las siguientes temáticas: 1) qué es un programa de rehabilitación, reubicación o reincorporación laboral, 2) responsabilidades ante el programa de rehabilitación, reubicación o reincorporación de las empresas, 3) descripción de los estándares normativos en los procesos de rehabilitación, reubicación o reincorporación laboral, 4) descripción de los procesos de rehabilitación, reubicación o reincorporación de las empresas y, 5) funciones de las ciencias médicas ante los procesos de rehabilitación, reubicación o reincorporación laboral. La última etapa correspondió a la realización final del documento a través de la validación de este.

Finalmente, el documento técnico resultante de este análisis documental, fue denominado "De regreso al trabajo", una herramienta de socialización de los procesos de rehabilitación y reincorporación laboral de los trabajadores en el sistema general de riesgos laborales, elaborada bajo un diseño práctico, técnico y llamativo, dirigido a gerentes y encargados del sistema de gestión de seguridad y salud en el trabajo, para facilitar la gestión administrativa frente a dichos procesos y la exigencia normativa.

\section{REFERENCIAS}

ARL Colpatria. (2015). Rehabilitación y retorno laboral. Recuperado de http://prevencionar.com. co/2015/10/21/rehabilitacion-y-retorno-laboral/ 
Fasecolda. (2016). Evolución de indicadores de riesgos laborales, segmentados por sectores económicos. Óscar Espinosa. Revista Fasecolda, (162), 48-59. Recuperado de https://revista.fasecolda.com/index.php/revfasecolda/article/view/201

Fundar. (2001). ¿Cómo hacer guías didácticas? Recuperado de http://www.fundacionarauco.cl/_file/file_ 3881_gu\%C3\%ADas\%20did\%C3\%A1cticas.pdf.

Ministerio de la Protección Social. (2005). Instrumentación del Manual Guía de Procedimientos de Rehabilitación y Reincorporación Ocupacional de los Trabajadores en el Sistema General de Riesgos Profesionales. Bogotá D. C., Colombia: Fundación GLARP - IIPD.

Ministerio de la Protección Social. (2010). Actualiæación Manual Guía sobre Procedimientos para la Rehabilitación y Reincorporación Ocupacional de los Trabajadores en el Sistema General de Riesgos Profesionales. Bogotá D. C., Colombia: Imprenta Nacional de Colombia.

Ministerio de Salud y Protección Social. (2015). Sala situacional de personas con discapacidad nacional.
Recuperado de http://discapacidadcolombia.com/ index.php/estadisticas

Ministerio del Trabajo. (2014). Plan Nacional de Seguridad y Salud en el Trabajo 2013-2021. Hacia una cultura preventiva. Recuperado de http://www.oiss. org/wp-content/uploads/2000/01/PlanNacionalDeSeguridadySaludEnElTrabajo.pdf

Ministerio del Trabajo. (2017). Resolución 1111, por la cual se definen los estándares mínimos del Sistema de Gestión de Seguridad y Salud en el Trabajo para empleadores y contratistas. DO 50189 (28 de marzo de 2017).

Organización Mundial de la Salud. (2017). 3 de diciembre 2017. Día Internacional de las Personas con Discapacidad. Recuperado de https:// www.paho.org/hq/index.php?option =com content\&view=article\&id=13967:a-day-for-all2017\&Itemid=72199\&lang=es

Universidad Autónoma Chapingo. (2009). Guía didáctica para la virtualización educativa en la Universidad Autónoma Chapingo. Recuperado de eduvirtual.chapingo.mx/archivos/guia_didactica.doc 